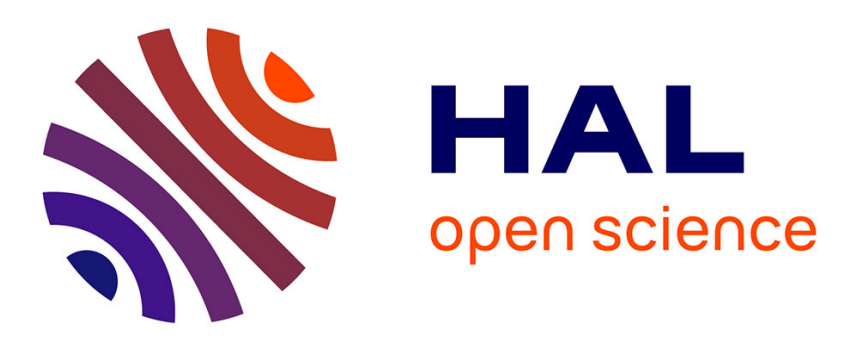

\title{
Frequency dependence of the UWB indoor propagation channel
}

Patrice Pajusco, Pascal Pagani

\section{To cite this version:}

Patrice Pajusco, Pascal Pagani. Frequency dependence of the UWB indoor propagation channel. EuCAP 2007: the second european conference on antennas and propagation, Nov 2007, Edinburgh, United Kingdom. hal-02316361

\section{HAL Id: hal-02316361 \\ https://hal.science/hal-02316361}

Submitted on 25 Oct 2019

HAL is a multi-disciplinary open access archive for the deposit and dissemination of scientific research documents, whether they are published or not. The documents may come from teaching and research institutions in France or abroad, or from public or private research centers.
L'archive ouverte pluridisciplinaire $\mathbf{H A L}$, est destinée au dépôt et à la diffusion de documents scientifiques de niveau recherche, publiés ou non, émanant des établissements d'enseignement et de recherche français ou étrangers, des laboratoires publics ou privés. 


\title{
FREQUENCY DEPENDENCE OF THE UWB INDOOR PROPAGATION CHANNEL
}

\author{
P. Pajusco*, P. Pagani ${ }^{\dagger}$ \\ * France Telecom / Orange Labs, 6 avenue des Usines, 90000 Belfort, France \\ † France Telecom / Orange Labs, 4 rue du Clos Courtel, 35510 Cesson Sévigné, France \\ Email: \{patrice.pajusco ; pascal.pagani\}@orange-ftgroup.com
}

Keywords: Ultra-wideband; radio wave propagation; channel characterization; frequency dependence.

\begin{abstract}
Ultra wideband (UWB) is seen as a major technology for future high rate, short range radio links. For the development of UWB-based communication systems for indoors, an accurate knowledge of the propagation channel is necessary. However, most of the available studies regarding the UWB radio channel concentrate on the lower frequencies of the FCC-defined spectrum mask, and experimental data is generally missing for the evaluation of the frequency dependence of the channel parameters. In this paper, an extensive sounding campaign is presented, where the UWB channel is probed from $3.1 \mathrm{GHz}$ to $11.1 \mathrm{GHz}$ in a typical indoor environment. Following a thorough analysis of the effect of the antennas on the measured data, a characterisation of the main propagation parameters is presented, with a specific interest on their frequency dependence. A frequency power decay close to the theoretical loss of $20 \mathrm{~dB}$ per decade is observed. Other typical parameters, such as the environment dependent path loss exponent, the delay spread or the power delay profile decay exponent show limited variations with increasing frequency. This behaviour may be explained by the characteristics of the main building materials constituting indoor environments, which present a relative stability in the frequency range of interest.
\end{abstract}

\section{Introduction}

UWB is a promising technology for short range telecommunication systems, consisting of transmitting signals over a wide frequency band, typically in the order of $500 \mathrm{MHz}$ to several $\mathrm{GHz}$ [1]. In 2002, the Federal Communications Commission (FCC) released its first Report and Order, allowing the use of UWB signals in the $3.1 \mathrm{GHz}-$ $10.6 \mathrm{GHz}$ band [2]. A number of applications are currently envisioned, providing high data rates for data transfer using low cost terminals ([1], [3]). A large amount of research on the physical layer has thus been done for a few years. In order to develop efficient transmission systems, one of the main issues is to establish a realistic propagation channel model. Such a model is expected to accurately reproduce the key effects of an UWB radio link, such as the temporal selectivity, the spatial and temporal fading, and the effect of people moving in the vicinity of the terminal.

UWB channel modelling is generally based on the analysis of experimental data, allowing for the characterization of the main channel parameters [4]. Accordingly, a number of UWB propagation experiments have been carried out since the appearing of this technology in the field of telecommunication ([5], [6]). Nevertheless, these propagation experiments are usually restricted to the lower part of the FCC-defined band. Only a few analyses focus on the whole UWB band extending from $3.1 \mathrm{GHz}$ to $10.6 \mathrm{GHz}$ ([7]-[10]). Thus, further investigations on the UWB channel are required, especially regarding the impact of the frequency.

This paper presents an experimental analysis of the influence of the frequency on the different characteristics of the UWB propagation channel. For this purpose, we studied the indoor radio channel over the global $3.1 \mathrm{GHz}-10.6 \mathrm{GHz}$ band, and on 7 partial bands of $528 \mathrm{MHz}$ each, which corresponds to the bandwidth selected for the UWB systems standard ECMA-368 [11]. Section 2 describes the experiment performed in a typical indoor environment. The influence of the antenna on the measurement results is then analysed in Section 3 . The two next sections are respectively dedicated to propagation loss results (Section 4) and to the study of large scale parameters in the delay domain (Section 5).

\section{Experiment description}

\subsection{General measurement setup}

Propagation channel measurement can be performed in either temporal or frequency domains. Due to the ease and advantages of its implementation, we chose a sounding technique in the frequency domain using a Vector Network Analyser (VNA) HP8510C. The Channel Transfer Function (CTF) $H(f)$ was thus obtained using standard $\mathrm{S}_{21}$ parameter measurements. The propagation channel was sampled over 4005 equally spaced frequencies between 3.1 and $11.1 \mathrm{GHz}$. Hence, the whole FCC-defined frequency band for indoor UWB applications was probed. This setup allowed a maximum excess delay of about $500 \mathrm{~ns}$ and a temporal resolution of about $125 \mathrm{ps}$.

Conical Monopole Antennas operating in the $1 \mathrm{GHz}$ $18 \mathrm{GHz}$ band (CMA 118) were used at each side of the radio link. The transmitting (Tx) antenna was mounted on a trolley to simplify the displacement to the different measurement locations. Depending on the configuration between the transmitter and the receiver, we used up to three Low Noise Amplifiers (LNA), with respective gains of $20 \mathrm{~dB}, 28 \mathrm{~dB}$ and $38 \mathrm{~dB}$. The three LNA present a constant gain over the measured frequency band, and their transfer function was subtracted from the channel measurement at the calibration stage. A step motor enabled the displacement of the Tx 


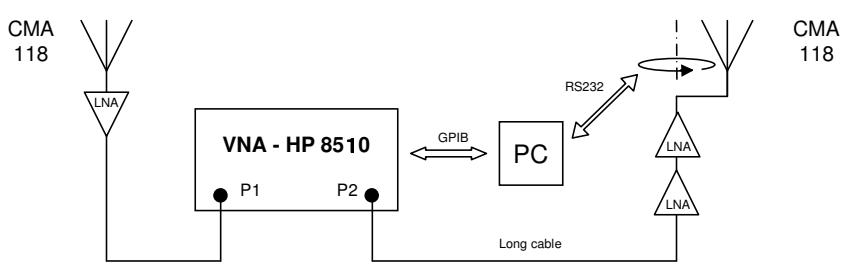

Figure 1: Schematic diagram of the measurement device.

antenna along a circle of $40 \mathrm{~cm}$ in diameter. For each measurement, 90 complex CTF were measured at regular intervals along the circular path. This setup enabled evaluating the local variations of the UWB propagation channel.

The receiving $(\mathrm{Rx})$ antenna was settled on a wall at a height of about 2 meters above the floor. Due to the asymmetry of the antenna pattern, the antenna was mounted upside down. This antenna was placed next to the VNA to minimise the loss of the received signal. The other port of the VNA was connected to the Tx antenna with a long and low loss cable. The maximum Tx-Rx distance was limited to $20 \mathrm{~m}$. The whole measurement process (calibration, antenna rotation, video capture and data saving) was automated using a specifically developed Matlab ${ }^{\mathrm{TM}}$ software. The schematic diagram of our measurements is depicted in Fig. 1.

\subsection{Environment description}

Measurements were performed in a typical indoor office environment, as depicted on Fig. 2. Two indoor office configurations were investigated. In the first scenario, the "access point" was located in a meeting room at a height of $2.19 \mathrm{~m}$. In the second one, the "access point" was located in a corridor at a height of $2.45 \mathrm{~m}$. In both cases, Line-Of-Sight (LOS) and Non Line-Of-Sight (NLOS) measurements were performed. The distance between transmitter and receiver varied from $1 \mathrm{~m}$ to $20 \mathrm{~m}$. The trolley supporting the Tx antenna was placed at more than 120 locations, of which one third corresponded to a LOS configuration and two thirds to a NLOS configuration. At each location, 90 measurements were performed using the rotating arm, leading to a total of over 10000 UWB CTF.

\subsection{Data processing}

During the propagation experiment, raw data from the VNA were stored in files. The measurement data was then calibrated with respect to a reference measurement where the emitter and the receiver were directly cable-connected. For measurements involving an LNA, the LNA transfer function was subtracted from the measurement at this stage. Several parameters were then extracted, by considering different bandwidths and central frequencies. First, the set of parameters was computed over the whole FCC-defined band $(3.1 \mathrm{GHz}-10.6 \mathrm{GHz})$. Then, the same set of parameters was computed over 7 partial bands of $528 \mathrm{MHz}$ each, which corresponds to the bandwidth specified by the UWB systems standard ECMA-368 [12]. The central frequencies of the selected bands extended from $4 \mathrm{GHz}$ to $10 \mathrm{GHz}$ with a $1 \mathrm{GHz}$ step. As a result, it was possible to analyse the effect of the

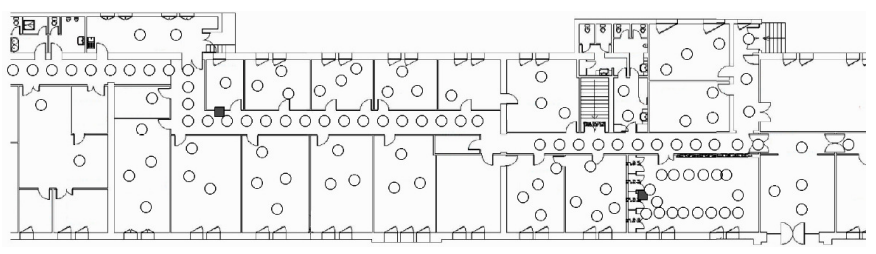

Corridor scenario

Meeting room scenario

Figure 2: Measurement locations for the UWB channel experiment. White circles represent emitter locations and shaded squares represent receiver locations.

central frequency on the characteristics of the UWB propagation channel. Most of computed parameters are recapped hereafter.

Each measurement being performed in the frequency domain, the measured quantity is the CTF $H(f)$. Note that the CTF represents the joint effects of the channel and of the antennas, the influence of the sounding equipment being removed through the calibration procedure. How the antenna effect is treated will be explained in details in Section 3.

The average Power Transfer Function (PTF) was obtained by averaging the power of different co-located CTF along the measurement circular path:

$$
\operatorname{PTF}(f)=\frac{1}{M} \sum_{m=1}^{M}\left|H_{m}(f)\right|^{2}
$$

where $M=90$ represents the number of co-located measurements, $m$ represents the measurement index and $H_{m}(f)$ is the corresponding CTF. Due to the CTF calibration, the PTF merely represents the average channel power attenuation for each measured frequency. The PTF was then used to compute the UWB Propagation Loss (PL) for a given central frequency $f_{c}$ in the measured band and a given observation bandwidth $B W$, according to the following formula.

$$
P L_{B W}\left(f_{c}\right)=\frac{1}{N} \sum_{f_{n}>f_{c}-\frac{B W}{2}}^{f_{n}<f_{c}+\frac{B W}{2}} P T F\left(f_{n}\right)
$$

where $N$ represents the number of measured frequencies $f_{n}$ in the considered bandwidth.

To compute time domain parameters, the Channel Impulse Response (CIR) $h(\tau)$ is obtained from the CTF using an inverse Fourier transform.. A Hanning window was applied at this stage to reduce the side lobe level. The Power Delay Profile (PDP) was deduced using a power average, as follows:

$$
P D P(\tau)=\frac{1}{M} \sum_{m=1}^{M}\left|h_{m}(\tau)\right|^{2}
$$

where the notations $M$ and $m$ are the same as in equation (1).

The decreasing slope of the PDP was fitted to the following exponential approximation, using a least squares method:

$$
P D P(\tau) \cong B^{2} \cdot e^{-\frac{\tau}{\gamma}}
$$

where $\gamma$ represents the PDP decay exponent, and $B$ is a normalising constant.

The mean delay $\tau_{m}$ and the delay spread $\tau_{R M S}$ were finally computed according to the following equations:

$$
\tau_{m}=\frac{\int_{-\infty}^{+\infty} \tau \cdot \operatorname{PDP}(\tau) d \tau}{\int_{-\infty}^{+\infty} P D P(\tau) d \tau}
$$




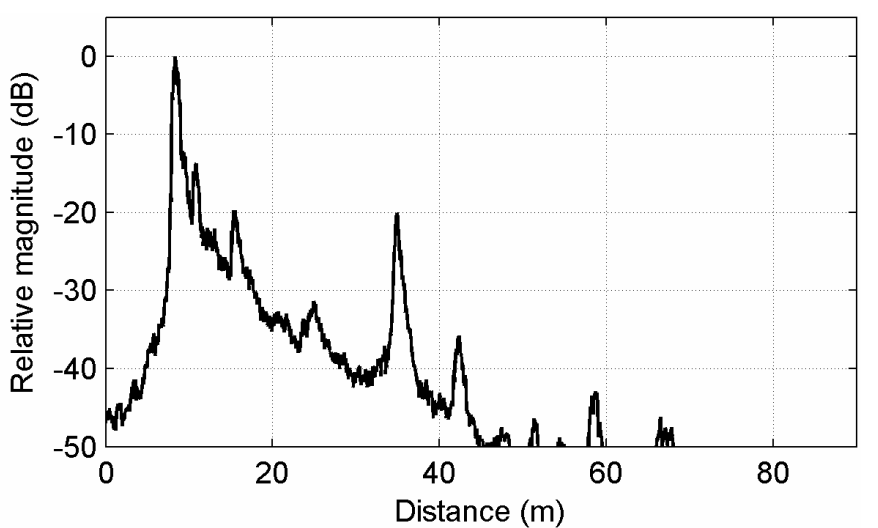

Figure 3: Example of average Power Delay Profile in LOS configuration.

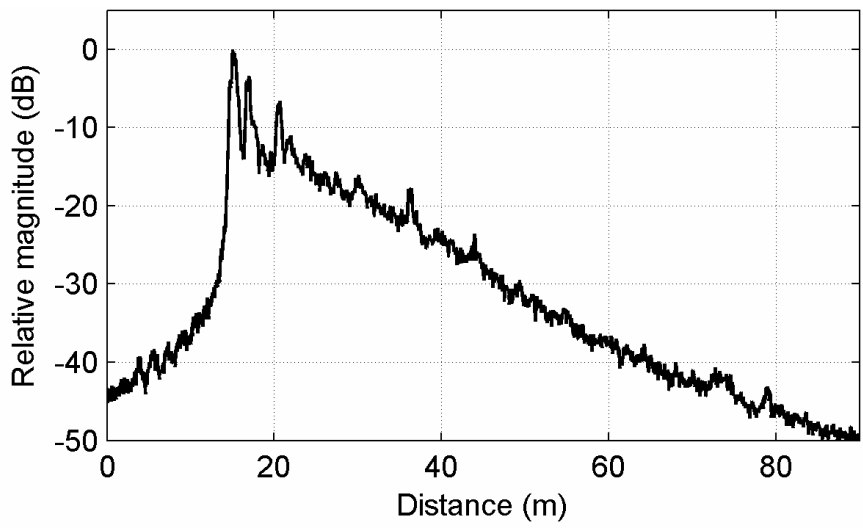

Figure 4: Example of average Power Delay Profile in NLOS configuration.

$$
\tau_{R M S}=\sqrt{\frac{\int_{-\infty}^{+\infty}\left(\tau-\tau_{m}\right)^{2} \cdot P D P(\tau) d \tau}{\int_{-\infty}^{+\infty} P D P(\tau) d \tau}}
$$

Figures 3 and 4 provide typical examples of measured PDP in LOS and NLOS configurations.

\section{Antenna analysis}

\subsection{General description}

When the radio channel is sounded over a frequency range representing more than an octave, the antenna may severely affect the measurements. The power received via each multipath impinging the Rx antenna depends directly on the antenna radiation pattern. Thus, if the antenna pattern is modified, the apparent channel properties may fluctuate accordingly. For instance, a narrow beam antenna focuses the transmitted energy on the direct path and the effect of the multipath components will therefore be attenuated. To minimize the influence of the antenna, we selected the CMA 118 antenna which presents an omni-directional radiation pattern and is vertically polarized. A picture of the antenna and its orientation are presented in Fig. 5.

In order to precisely assess the effect of the antennas used for the experiment, both $\mathrm{Tx}$ and $\mathrm{Rx}$ antennas were characterized in 3 dimensions (3D) in an anechoic chamber. The radiation
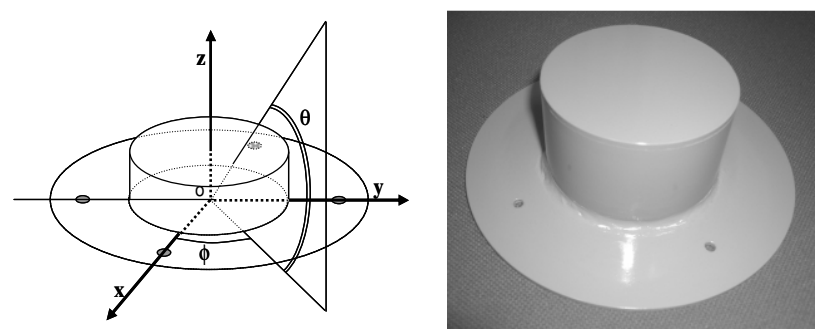

Figure 5: Reference axis and picture of the antenna CMA 118.

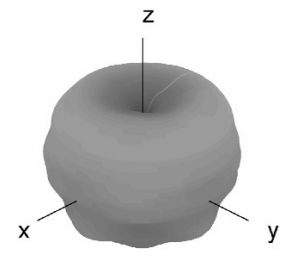

(a)

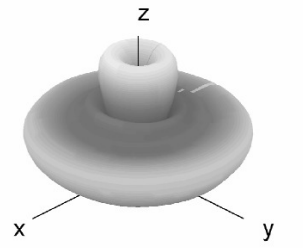

(b)

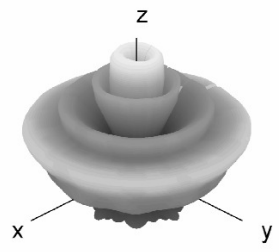

(c)
Antenna gain $(\mathrm{dBi})$

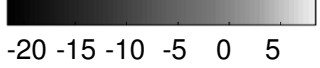

Figure 6: Examples of measured radiation patterns of the antenna CMA 118 at $3 \mathrm{GHz}$ (a), $7 \mathrm{GHz}$ (b) and $10 \mathrm{GHz}$ (c).

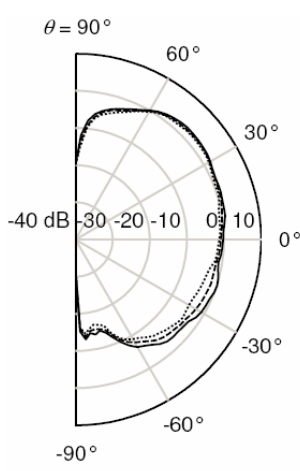

(a)

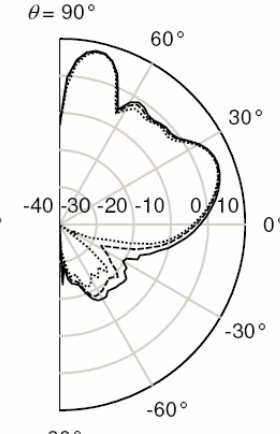

$-90^{\circ}$

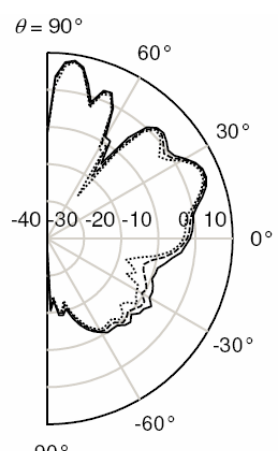

$-90^{\circ}$

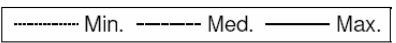

(b) (c)
Figure 7: Dispersion of the antenna CMA 118 radiation pattern in azimuth. Minimum, median and maximum antenna gain in azimuth for each elevation $\theta$, at $3 \mathrm{GHz}$ (a), $7 \mathrm{GHz}(b)$, and $10 \mathrm{GHz}(c)$.

patterns of the two antennas are quite similar. However, in spite of the high quality of this product in terms of gain flatness over the measurement bandwidth, we observed a noticeable variation of the antenna characteristics as the frequency increases. As an example, Fig. 6 depicts the 3D antenna pattern measured at three selected frequencies within the FCC-defined frequency band. We can notice that the radiation pattern becomes less and less omni-directional in the elevation plane as the frequency increases. To evaluate the omni-directional behaviour of the antenna in the horizontal plane, we computed the minimum, the median and the maximum antenna gain in azimuth for each elevation. Figure 7 depicts the results obtained for the three frequencies taken as examples in Fig. 6. One can observe that the radiation pattern is relatively constant in azimuth for a given elevation and a given frequency. 


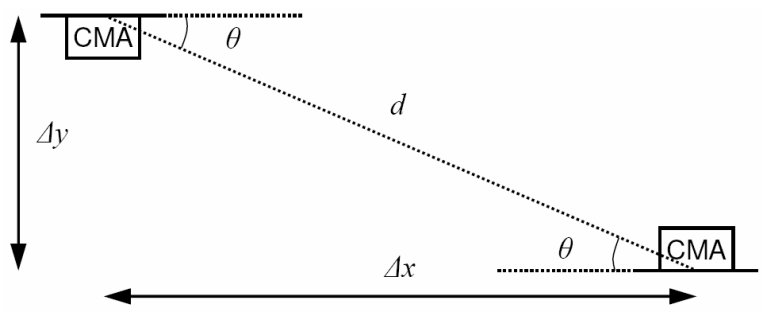

Figure 8: Antennas configuration during the experiment. $\Delta x$, $\Delta y$ and $d$ respectively represent the horizontal, vertical and effective distances between the antennas, and $\theta$ is the elevation angle of the direct path.

\subsection{Influence of the antennas on path loss}

\section{a) Distance domain}

To evaluate the influence of the antenna on the distance dependent path loss, we consider a typical LOS configuration, as depicted in Fig. 8. In a typical sounding experiment, we normally expect a more severe channel attenuation when the $\mathrm{Tx}-\mathrm{Rx}$ distance increases. However, an increasing distance also leads to a decrease of the elevation angle $\theta$. Hence, the observed attenuation is not only due to changes in the length of the direct path, but also to variations of the antenna pattern with elevation. In our experiment, measurements were performed for distances $\Delta x$ varying from $1 \mathrm{~m}$ to $20 \mathrm{~m}$. With a height difference $\Delta y$ of about $90 \mathrm{~cm}$, the elevation $\theta$ varied between $3^{\circ}$ and $42^{\circ}$. Figure 7 shows for three demonstrative frequencies that in this region, the radiation pattern presents small, but not negligible fluctuations. Depending on the Tx$\mathrm{Rx}$ distance and on the frequency, the global variation of the direct path power due to the $\mathrm{Tx}$ and $\mathrm{Rx}$ antennas may be significant, in the order of $10 \mathrm{~dB}$. Thus, the effect of the nonideal antenna needs to be removed from the channel measurement. Otherwise, the antennas may introduce apparent fluctuations in the path loss parameters.

\section{b) Frequency domain}

In an ideal, free-space configuration, the propagation loss $P L$ in $\mathrm{dB}$ between the transmitted and received power is given by Friis' formula:

$$
P L(f, d)=20 \cdot \log \left(\frac{4 \pi \cdot f \cdot d}{c}\right)-G_{T x}(f)-G_{R x}(f)
$$

where $f$ represents the frequency in $\mathrm{Hz}, d$ represents the $\mathrm{Tx}-$ $\mathrm{Rx}$ distance in $\mathrm{m}, G_{T x}$ and $G_{R x}$ represent the $\mathrm{Tx}$ and $\mathrm{Rx}$ antenna gains in $\mathrm{dBi}$, and $c$ is the speed of light. Equation (7) implies that for a given distance $d$, the channel average PTF (in linear scale) should be proportional to $f^{-2}$. Noteworthy frequency power decay has been observed on previous UWB channel measurements $([8,9,13])$. In [8], the channel power attenuation was observed to be proportional to $f^{-k_{f}}$, with $k_{f}$ varying between 1.6 and 2.8. Reference [9] proposed a rather different approach, by fitting the measured PTF in $\mathrm{dB}$ to an exponential law.

When considering frequency power decay, one should keep in mind that the frequency term $20 \cdot \log (f)$ in Friis' formula is related to the effective aperture of an ideal, isotropic antenna,

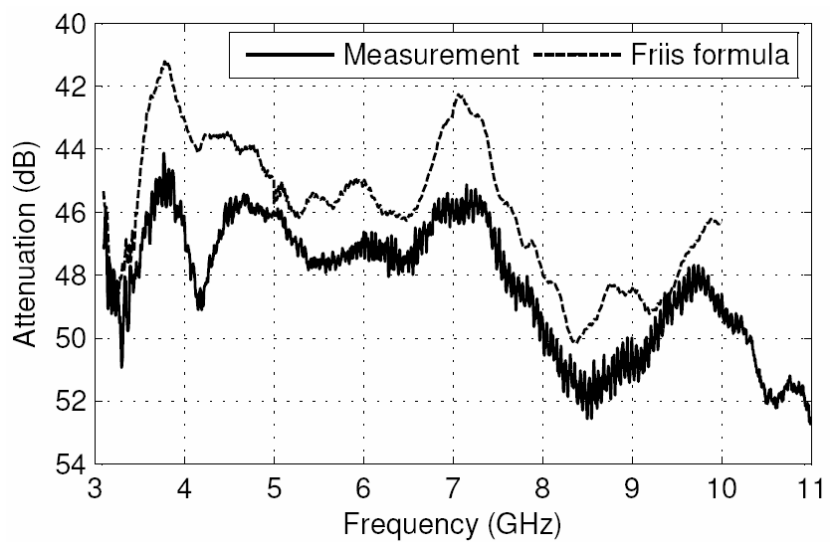

Figure 9: Measured PTF compared to Friis' formula.

while the characteristics of the real antennas are taken into account in the gains $G_{T x}$ and $G_{R x}$. PTF measurements performed with non-ideal antennas are hence highly sensitive to the antenna patterns. Figure 9 illustrates this dependency by representing a PTF measured in a LOS configuration, along with the theoretical path loss computed from equation (7), where the gains $G_{T x}$ and $G_{R x}$ were measured in an anechoic chamber at an elevation corresponding to the measurement situation. One can observe the predominant effect of the antennas, as the general shape of the measured PTF faithfully follows the variations of the antenna gains. Again, this result shows that the effects of non-ideal antennas should be taken into account prior to the computation of the channel characteristics.

\section{Propagation loss results}

\subsection{Frequency dependent path loss}

In order to assess the frequency dependent power decay linked to a channel using theoretical isotropic antennas, we studied the experimental values of the channel path loss at regularly spaced frequencies between $4 \mathrm{GHz}$ and $10 \mathrm{GHz}$. For each measurement location, the pathloss $P L(f, d)$ was extracted at the frequencies of interest from the measured PTF. In order to remove the distance dependency, each PTF was initially normalised, by arbitrarily setting the attenuation of the total received power over the whole measurement bandwidth to $0 \mathrm{~dB}$. The resulting normalised path loss $P L_{\text {norm }}(f)$ could then be fitted to a linear approximation of the type:

$$
P L_{\text {norm }}(f)=P L_{\text {norm }}\left(f_{0}\right)+10 \cdot k_{f} \cdot \log \left(\frac{f}{f_{0}}\right)+S(f)
$$

where $k_{f}$ represents the frequency power decay exponent, $f_{0}$ is the central frequency and $S(f)$ accounts for the fluctuations of the experimental data around the linear approximation.

The normalised path loss averaged over all measurement locations is represented against frequency in Fig. 10. At first, no antenna correction was applied (black dots). One may note an increase of the path loss with increasing frequency, but there is no way to distinguish between the frequency dependent power decay and variations due to the antenna pattern. The computed power decay exponent is $k_{f}=2.60$, 


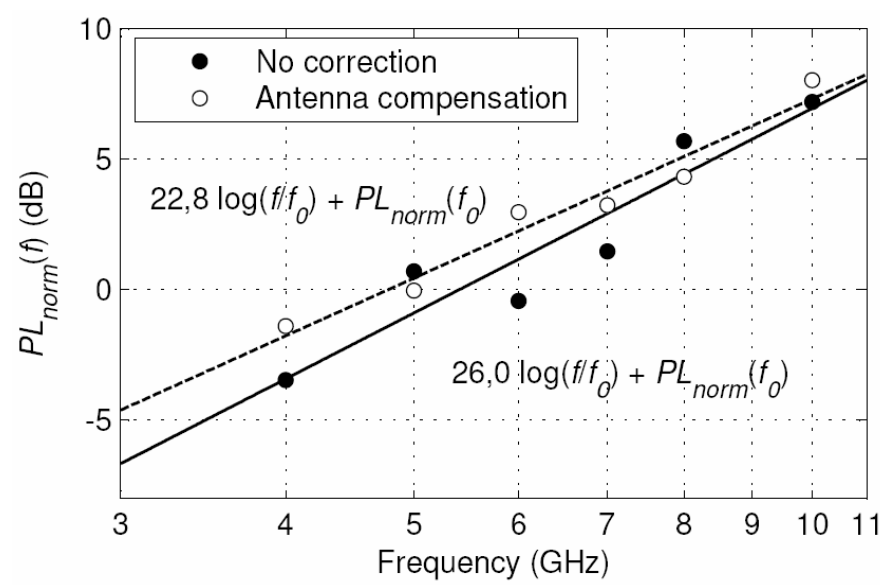

Figure 10: Average normalised path loss vs. frequency.

leading to a standard deviation of the measured data around the linear approximation of $1.2 \mathrm{~dB}$. Secondly, the measured antenna gains $G_{T x}(f)$ and $G_{R x}(f)$ were subtracted from the measured PTF at each frequency of interest prior to path loss calculation (white dots). The antenna gain was selected by taking the direction of the direct Tx-Rx path into account. This approach may seem simplistic, as the total power is not received via the direct path only, but it allows a sensible compensation of the antenna effect, leading to satisfactory results: in this case, the dispersion of the measured plots around the linear approximation is reduced to a standard deviation of $0.6 \mathrm{~dB}$. The frequency dependent power decay $k_{f}$ falls to 2.28. With respect to the error level inherent to the measurement and computation methods, this value may be considered close to the theory. Hence, we recommend using the theoretical frequency $\operatorname{loss}$ of $20 \cdot \log (f)$ in the modelling of UWB channels, which correspond to the theoretical effect of ideal, isotropic antennas.

\subsection{Distance dependent path loss over the FCC band}

In this paragraph, we analyse the propagation loss parameters on the global FCC-defined frequency band extending from $3.1 \mathrm{GHz}$ to $10.6 \mathrm{GHz}$. As observed in Section 4.1, a frequency $\operatorname{loss}$ of $20 \cdot \log (f)$ accounting for ideal, isotropic antennas may be assumed. Section 3 also pointed out that the real antennas used during the experiment could adversely affect propagation loss analyses in both frequency and distance domains. Hence, the collected data was corrected to the possible extent, by removing the gain of both antennas, measured at the central frequency of the analysed band, with an angular elevation corresponding to the direction of the TxRx path. Note that for the global UWB frequency band, the measurement performed at each frequency was corrected using the nearest measured radiation pattern. The resulting path loss in $\mathrm{dB}$ was fitted to the general formula in the form:

$$
\begin{aligned}
P L(f, d)=P L\left(f_{0}, d_{0}\right) & +20 \cdot \log \left(\frac{f}{f_{0}}\right) \\
& +10 \cdot k_{d} \log \left(\frac{d}{d_{0}}\right)+S(d)
\end{aligned}
$$

where $k_{d}$ is the environment dependent path loss exponent, $d_{0}$

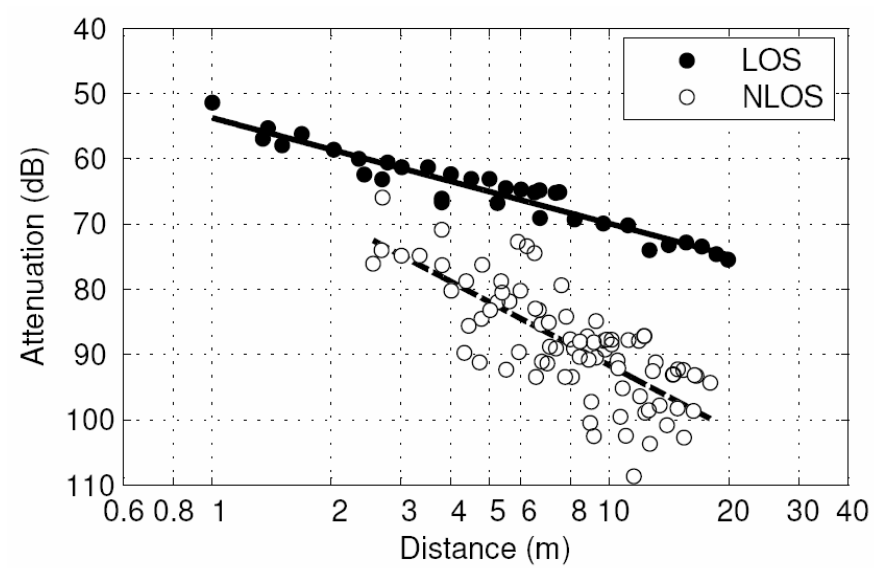

Figure 11: Scatter plot of the power attenuation vs. distance.

is an arbitrary distance of $1 \mathrm{~m}, f_{0}$ is the central frequency of $6.85 \mathrm{GHz}$, and $S$ is the shadowing in $\mathrm{dB}$, with zero mean and standard deviation $\sigma_{S}$.

Figure 11 depicts the path loss results for the global UWB frequency band, i.e. $3.1 \mathrm{GHz}-10.6 \mathrm{GHz}$. The measurement plots mainly follow a linear decay in log-scale, which corresponds to an exponential decay of the received power with respect to the distance. The parameters $k_{d}, P L\left(f_{0}, d_{0}\right)$ and $\sigma_{S}$ were computed by linear regression using a least squares method. In the LOS case, a path loss exponent $k_{d}=1.62$ was recorded, with a standard deviation $\sigma_{S}=1.7 \mathrm{~dB}$. In the NLOS case, the measurement plots are somewhat more dispersed, leading to a value of $k_{d}=3.22$, with a standard deviation $\sigma_{S}=5.7 \mathrm{~dB}$. These values are slightly lower but in the range of the UWB path loss exponents computed from other measurements in an indoor environment $[6,7]$. The value of $P L\left(f_{0}, d_{0}\right)$ was respectively evaluated at $53.7 \mathrm{~dB}$ in the LOS case and $59.4 \mathrm{~dB}$ in the NLOS case.

\subsection{Frequency dependence of the path loss parameters}

A few results only are available in the literature about the frequency dependence of the path loss exponent. An increase of $k_{d}$ with frequency is suggested in the NLOS case in [14]. Reference [11] reports a marked increase of the path loss exponent in the LOS case, while NLOS measurements exhibit the opposite trend. Hence, more experimental analysis was required on this subject.

Table 1 summarizes the values obtained for $k_{d}, \sigma_{S}$ and $P L\left(f_{0}, d_{0}\right)$ when derived from the seven partial bands of $528 \mathrm{MHz}$ each. The path loss exponent $k_{d}$ varies between 1.44 and 1.92 in the LOS case and between 2.82 and 4.21 in the NLOS case. As a global observation, the path loss exponent is relatively stable. Slightly higher values may be detected at high frequencies $(8 \mathrm{GHz}$ to $10 \mathrm{GHz}$ ). This small increase may arise from a variation in the characteristics of the building material in the vicinity of the radio link, but no regular trend is observable. The parameters $\sigma_{S}$ and $P L\left(f_{0}, d_{0}\right)$ do not appear to be affected by the central frequency of the observed band. Measurement results presented in [15] indicate that the complex permittivity of concrete and plasterboard are relatively stable over the whole band defined for UWB communications. Similarly, reference [16] reports reasonably 


\begin{tabular}{|c|c|c|c|c|c|c|c|}
\hline \multirow{2}{*}{$\begin{array}{c}\text { Band- } \\
\text { width } \\
(\mathrm{GHz})\end{array}$} & $\begin{array}{c}\text { Center } \\
\text { freq. } \\
(\mathrm{GHz})\end{array}$ & \multicolumn{3}{|c|}{ LOS } & \multicolumn{3}{c|}{ NLOS } \\
\cline { 3 - 8 } & $k_{d}$ & $\begin{array}{c}\sigma_{S} \\
(\mathrm{~dB})\end{array}$ & $\begin{array}{c}P L_{0} \\
(\mathrm{~dB})\end{array}$ & $k_{d}$ & $\begin{array}{c}\sigma_{S} \\
(\mathrm{~dB})\end{array}$ & $\begin{array}{c}P L_{0} \\
(\mathrm{~dB})\end{array}$ \\
\hline 0.528 & 4 & 1.44 & 2.2 & 54.9 & 2.93 & 5.5 & 60.9 \\
\cline { 2 - 8 } & 5 & 1.63 & 2.6 & 52.3 & 3.12 & 5.7 & 58.5 \\
\cline { 2 - 8 } & 6 & 1.50 & 2.8 & 55.1 & 2.82 & 6.1 & 62.9 \\
\cline { 2 - 8 } & 7 & 1.51 & 2.6 & 53.2 & 2.93 & 5.7 & 61.2 \\
\cline { 2 - 8 } & 8 & 1.81 & 3.6 & 51.2 & 3.43 & 6.2 & 58.1 \\
\cline { 2 - 8 } & 9 & 1.92 & 2.3 & 53.5 & 4.21 & 6.9 & 55.9 \\
\hline \hline 7.5 & 6.85 & 1.62 & 1.7 & 53.7 & 3.22 & 5.7 & 59.4 \\
\hline
\end{tabular}

Table 1: Distance dependent path loss parameters for different frequency bands.

low variations of the transmission loss measured for concrete and wood between the lower and upper frequencies of the UWB spectrum (less than $2 \mathrm{~dB}$ ). These building materials constitute most of the walls in our measured environment. It is thus not surprising to observe a relative stability in the propagation properties of the UWB radio channel with respect to the frequency. Furthermore, one should note that all measurements were performed on a single floor. Inter-floor measurements involve possible wave propagation through reinforced concrete slabs, where the regular metallic netting could introduce frequency dependencies in the received signal. This was not the case in our experiment.

\section{Large-scale delay domain results}

\subsection{Delay spread}

The delay spread $\tau_{R M S}$ was computed from each collected PDP, over the whole FCC-defined band $(3.1 \mathrm{GHz}-$ $10.6 \mathrm{GHz}$ ). A threshold of $20 \mathrm{~dB}$ below the maximum value of the PDP was used to select the significant delays only and avoid the effect of noise. Over all measurement locations in a LOS configuration, the average value of the delay spread was $\tau_{R M S}=4.1 \mathrm{~ns}$ with a standard deviation $\sigma_{\tau}=2.7 \mathrm{~ns}$. In the NLOS configuration, the average delay spread was $\tau_{R M S}=9.9 \mathrm{~ns}$ with a standard deviation $\sigma_{\tau}=5.0 \mathrm{~ns}$. These figures agree with the values generally reported in the literature on UWB measurements [6].

In order to observe the evolution of these parameters with frequency, the average value of $\tau_{R M S}$ computed for seven partial bands of $528 \mathrm{MHz}$ each are plotted against central frequency in Fig. 12. For each band, the value of $\sigma_{\tau}$ is represented by the length of the vertical line. No frequency dependence of the RMS delay spread is noticeable. The values of $\tau_{R M S}$ and $\sigma_{\tau}$ obtained in each $528 \mathrm{MHz}$-wide frequency band are relatively close to the values observed for the global band extending from $3.1 \mathrm{GHz}$ to $10.6 \mathrm{GHz}$.

\subsection{Power Delay Profile}

Figures 3 and 4 present typical PDPs in LOS and NLOS configurations. Note that for the convenience of path

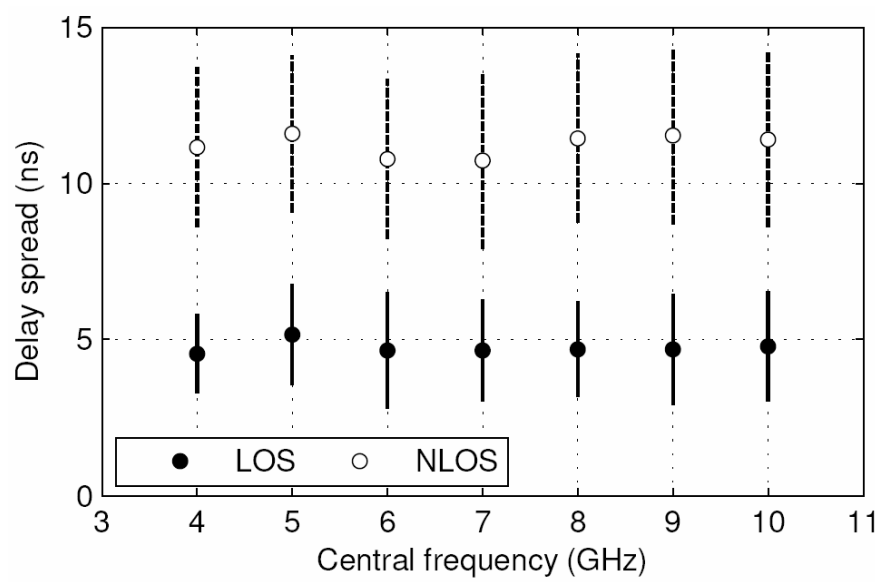

Figure 12: Average RMS delay spread and standard deviation vs. central frequency.

interpretation, the delay on the horizontal axis has been converted in path length in meters. In both LOS and NLOS cases, one may observe one or several clusters, corresponding to a main path followed by an exponential decay of diffuse power. In the LOS case, walls or pieces of furniture in the vicinity of the radio link produce strong reflected or diffracted echoes, which explains the presence of peaks in the PDP. The global shape of the PDP is generally smoother in the NLOS case, however multiple clusters may occasionally appear.

The slope of the PDP exponential decay has been computed for each measurement location as explained in section 2.3. The decay exponent $\gamma$ was separately computed for the whole FCC-defined band and for the seven partial bands of $528 \mathrm{MHz}$ width each. Over the global band ranging from 3.1 $\mathrm{GHz}$ to $10.6 \mathrm{GHz}$, the average value of the decay exponent was $\gamma=15.1 \mathrm{~ns}$ with a standard deviation of $\sigma_{\gamma}=3.8 \mathrm{~ns}$. From the wide range of experimentally observed values $[6,7]$, this parameter seems highly environment dependent, and more research should be concentrated on this issue.

Figure 13 presents the average values of the PDP decay exponent obtained for seven bands of $528 \mathrm{MHz}$ each, with different central frequencies. The corresponding standard deviations are represented by the length of the vertical lines. Clearly, there is no dependence of the decay exponent on the central frequency of each band, as the observed values are similar for all considered partial bands.

\section{Conclusion}

In this paper, we presented an exhaustive measurement campaign designed in the $3.1 \mathrm{GHz}-11.1 \mathrm{GHz}$ frequency band to characterise the UWB indoor propagation channel. The main purpose was to evaluate the impact of frequency on the channel main parameters. Using a VNA and two antennas CMA 118, over 10000 channel transfer functions were collected in both LOS and NLOS configurations. Despite the high quality of the antenna specifications in terms of gain flatness across the band, we demonstrated that the non-ideal antenna pattern could adversely affect the measured data in both frequency and distance domains. Hence, the antenna characteristics need to be precisely measured and removed to the possible extent from the raw collected data. This 
technique was validated through the computation of the frequency dependent power decay: after correction of the antenna effects, the channel frequency loss was shown to approach the theoretical loss of $20 \mathrm{~dB}$ per decade. On a modelling point of view, this corresponds to using ideal, isotropic antennas. The effect of the practical antennas with respect to this theoretical case should be modelled separately, and added in the simulation chain for a realistic reproduction of the global radio link.

The main channel parameters were extracted over the global FCC-defined frequency band, extending from $3.1 \mathrm{GHz}$ to 10.6 GHz. A decrease of the received power with increasing distance was observed, with an average path loss exponent of 1.62 in the LOS case and 3.22 in the NLOS case. The measured PDP generally presented a clustering of the multipath components, with respective average delay spreads of $4.1 \mathrm{~ns}$ and $9.9 \mathrm{~ns}$ in the LOS and NLOS cases, and an average decay exponent of $15.1 \mathrm{~ns}$. When computed from several partial bands of $528 \mathrm{MHz}$ each at different central frequencies, these parameters showed no or little impact of the frequency. A slight increasing trend of the path loss exponent with increasing frequency could be observed, but this topic needs further research for validation. As a general observation, a constant behaviour of the channel may be assumed over the whole UWB frequency band, in addition to the abovementioned frequency power decay. Using the adequate frequency dependent path loss formula, models based on measurements performed in the lower part of the UWB spectrum could thus be easily generalised to higher central frequencies. This conclusion comes in line with published results on the transmission coefficients of the main building material in indoor environments, which presented a relative stability across the FCC-defined frequency spectrum for UWB devices.

\section{References}

[1] L. Yang and G. B. Giannakis, "Ultra-wideband communications: an idea whose time has come", IEEE Signal Processing Magazine, 21, pp. 26-54, (2004).

[2] FCC, "First report and order, revision of Part 15 of the Commission's rules regarding ultra-wideband transmission systems", FCC ET Docket 98-153, (2002).

[3] D. Porcino and W. Hirt, "Ultra-wideband radio technology: potential and challenges ahead", IEEE Communications Magazine, 41, pp. 66-74, (2003).

[4] F. Molisch, J. R. Foerster, and M. Pendergrass, "Channel models for ultrawideband personal area networks", IEEE Wireless Communications, 10, pp. 14-21, (2003).

[5] Z. Irahhauten, H. Nikookar, and G. J. M. Janssen, “An overview of ultra wide band indoor channel measurements and modeling", IEEE Microwave and Wireless Components Letters, 14, pp. 386-388, (2004).

[6] F. Molisch, K. Balakrishnan, C. Chong, et al., "IEEE 802.15.4a channel model - final report", IEEE P802.15 Working Group for WPANs, Technical Report IEEE P802.15-04/0662r1-SG4a, (2004).

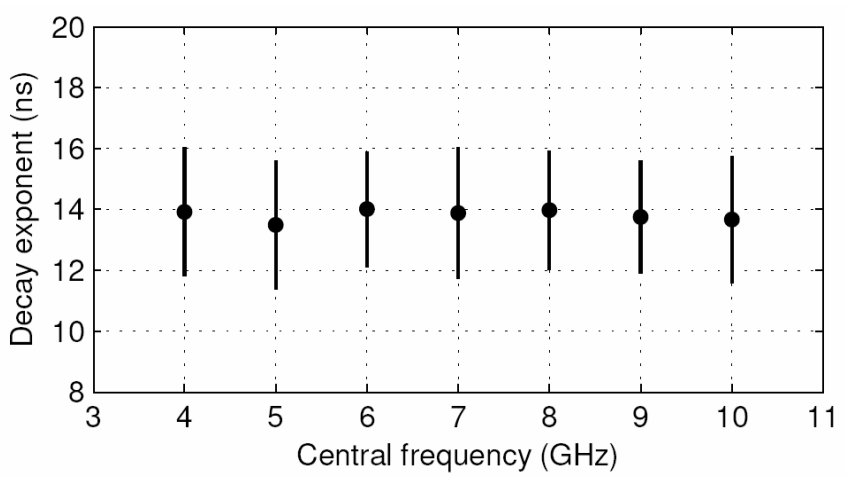

Figure 13: Average decay exponent and standard deviation vs. central frequency.

[7] J. Kunisch and J. Pamp, "Measurement results and modeling aspects for the UWB radio channel", IEEE Ultra Wideband Systems and Technologies, Baltimore MD, USA, (2002).

[8] Alvarez, G. Valera, M. Lobeira, et al., "New channel impulse response model for UWB indoor system simulations", IEEE Vehicular Technology Conference, Seoul, Korea, (2003).

[9] R. M. Buehrer, W. A. Davis, A. Safaai-Jazi, et al., "Characterization of the ultra-wideband channel", IEEE Ultra Wideband Systems and Technologies, Reston VA, USA, (2003).

[10] D. Cassioli, A. Durantini, and W. Ciccognani, "The role of path loss on the selection of the operating bands of UWB systems", IEEE International Symposium on Personal, Indoor and Mobile Radio Communications, Barcelona, Spain, (2004).

[11] ECMA International, "High Rate Ultra Wideband PHY and MAC Standards", standard no. ECMA-368, (2005).

[12] D. Cheung and C. Prettie, "A Path Loss Comparison Between the $5 \mathrm{GHz}$ UNII Band (802.11a) and the 2.4 GHz ISM Band (802.11b)", Intel Corporation Technical Document, (2002).

[13] B. M. Donlan, S. Venkatesh, V. Bharadwaj, et al., "The ultra-wideband indoor channel", IEEE Vehicular Technology Conference, Milan, Italy, (2004).

[14] R.-R. Lao, J.-H. Tarng, and C. Hsiao, "Transmission coefficients measurement of building materials for UWB systems in 3-10 GHz", IEEE Vehicular Technology Conference, Seoul, Korea, (2003).

[15] Muqaibel, A. Safaai-Jazi, A. Bayram, et al., "Ultra wideband material characterization for indoor propagation", IEEE Antennas and Propagation Society International Symposium, Columbus OH, USA, (2003).

[16] P. Pagani, P. Pajusco, and S. Voinot, "A Study of the Ultra-Wide Band Indoor Channel: Propagation Experiment and Measurement Results", International Workshop on Ultra Wideband Systems, Oulu, Finland, (2003).

[17] S. S. Ghassemzadeh, R. Jana, C. W. Rice, et al., "A statistical path loss model for in-home UWB channels", IEEE Conference on Ultra Wideband Systems and Technologies, Baltimore MD, USA, (2002). 